\title{
The Acute Effect of Proprioceptive Neuromuscular Facilitation in Explosive Force and Jump Resistance of Volleyball Players
}

\author{
Dao Chanh Thuc* \\ Department of Physical Education, An Giang University, Vietnam
}

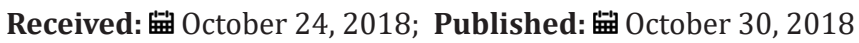

*Corresponding author: Dao Chanh Thuc, Department of Physical Education, An Giang University, Vietnam

\begin{abstract}
Stretching during warm-up has been widely discussed for exerting a possible influence on performance. Thus, the purpose of this study was to verify the influence of the Proprioceptive Neuromuscular Facilitation (PNF) in the explosive force (EF) and the jump resistance (JR) of athletes. Twelve male volleyball players (21 \pm 4 years; $178 \pm 0 \mathrm{~cm} ; 75.82 \pm 11.02 \mathrm{~kg} ; 23 \pm 2.87 \mathrm{~kg} / \mathrm{m} 2)$ underwent two PNF protocols: a) Protocol 1: four repetitions with 5- second maximum voluntary isometric contraction followed by 30 seconds of passive stretching and b) Protocol 2: four repetitions with 5-second maximum voluntary isometric contraction followed by 60 seconds of passive stretching. For the assessment of EF and JR the height of the countermovement vertical jump was considered measured through the Jump System Pro. The Student t test was used to compare the heights of the jumps between pre and post stretching moments for the two analyzed variables. EF (Protocols 1 and $2 \mathrm{t}(7)=1.462, \mathrm{p}=0.261$, and $\mathrm{t}(7)=1.402$, $\mathrm{p}=0.302$, respectively) and JR (Protocols 1 and 2, t (7) $=1.542, \mathrm{p}=0.242$, and t $(7)=0542, \mathrm{p}=0.712$, respectively) did not differ significantly $(p>0.05)$ after both stretching protocols. The results of this study show that the stretching protocols used did not acutely affect the performance of EF and JR of volleyball athletes.
\end{abstract}

Keywords: Stretching; Performance; Athletes; Sport; Volleyball

\section{Introduction}

The inclusion of stretching during warming up is a common practice within the training sessions of professional and recreational athletes [1,2]. Historically, this practice was maintained since it enforced possible effects on improved performance and injury prevention [3-5]. However, more recent studies have challenged these ideas, demonstrating that stretching before the main part of the training session does not prevent exercise-related injuries, and besides, depending on its type, intensity and volume, it can promote temporary decreases in neuromuscular performance and reduction of performance $[2,6,7]$.

In relation to the reduction in performance, the effect of several stretching methods has been verified in the manifestations of the force [6]. Among the types of stretching, the static is the most studied and the one that presents greater consensus among the results about the performance. In contrast, the Proprioceptive Neuromuscular Facilitation (PNF) still presents gaps regarding its effect on performance. These manifestations, the explosive force, especially of the lower limbs, is one of the most important factors that determine the physical condition of the athlete and their performance [8], especially when this ability is inherent to the sport, that can be affected by stretching. Another important indicator of the athlete's physical performance is muscular endurance that can also be affected by stretching protocols performed during the warming up phase of a training session $[9,10]$.

The volleyball is a sport that requires constant jumps $[5,11]$ requiring that players have the ability to produce explosive force of lower limbs as well as have the ability to maintain the performance of these jumps (jump resistance) during a match. Especially in a real competitive game situation, the performance of numerous jumps within a match [12] demonstrates the need to maintain a high level of explosive force in this sport mode. Knowing the importance of maintaining these manifestations of force for success in the sport, it is essential that coaches have knowledge of factors that can impair the performance of the athletes.

Some studies have shown that stretching can negatively influence the physical performance of athletes by generating decreased in muscle strength [13]. Thus, the effect of stretching on performance has been widely discussed [1]. Among the types 
of stretching, the static is the one that presents the most studies and that show agreeable data that discourages its conduction before strength and power activities [1,2]. The Proprioceptive Neuromuscular Facilitation (PNF) is a fairly common type of stretching in the athletic scenario [14], however, unlike static stretching, it presents limited evidence about its effect on the manifestations of force [1,2].

The few studies that sought to verify the influence of PNF in performance show very divergent results [9]. Specifically, regarding the explosive force measured from the performance in vertical jumps, there are studies that showed negative effect $[2,13]$ and others which did not find performance reduction after PNF protocol $[15,16]$. Regarding the muscle endurance, Franco and Colleagues [10] and Barroso and Colleagues [9] evaluated muscle resistance through the number of repetitions performed in the exercises of bench press and leg press, respectively, and found reductions of the number of repetitions after PNF, showing that such type of stretching can negatively influence muscle resistance. However, to our knowledge, no study analyzed the effect of PNF on jump resistance (JR).

Based on this controversy regarding the acute effect of stretching on performance and, therefore, considering that the PNF is a common type of stretching however without agreement results in the literature regarding performance, the aim of this work was to analyze the acute effect of PNF on the explosive force (EF) and jump resistance (JR) of volleyball athletes. Considering the significant body of evidence that shows the negative effect of passive stretching in performance and knowing that it composes the elongation of the PNF type, our initial hypothesis is that stretching applied in this study exerts negative influence on the performance of explosive force and muscular endurance, the reduction of performance dependent on the protocol used.

\section{Material and Methods}

Twelve male athletes participated in the study $(21 \pm 4$ years old; $178 \pm 0 \mathrm{~cm} ; 75.82 \pm 11.02 \mathrm{~kg} ; 23 \pm 2.87 \mathrm{~kg} / \mathrm{m}^{2}$ ) from the university volleyball team. They all had routines of at least four workouts per week with an average duration of 90 mins per session. It was adopted as exclusion criterion the athlete who reported recent lower limb injury or missing any of the sessions. An athlete was excluded on account of injury in one of the lower limbs.

Each athlete has been to the laboratory in three different moments separated for at least 48 hours of rest and at the same time of day. The first session was intended to familiarize the subjects with the procedures and equipment. During the other two days the experimental sessions with stretching occurred. Prior to all sessions, a five-minute warm up was held, with a progressive intensity race and specific volleyball moves. The structure of the sessions is described in Figure 1.

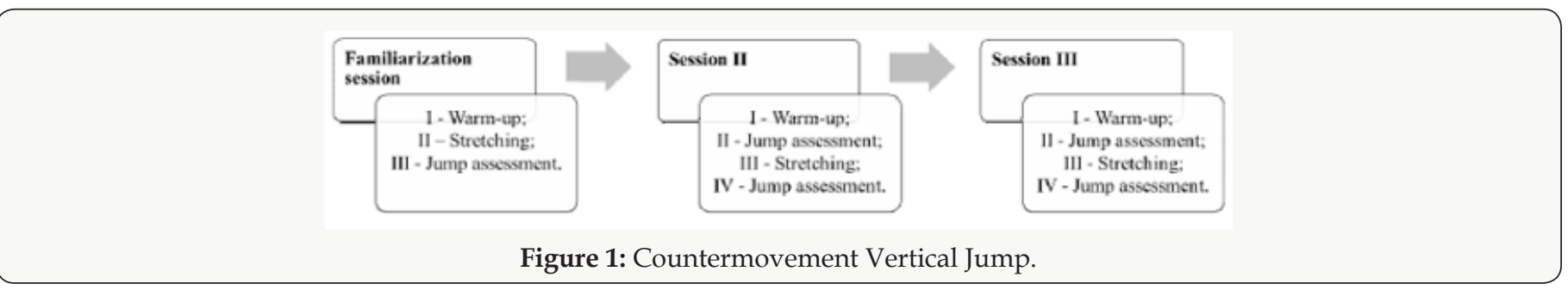

\section{Countermovement Vertical Jump}

For the evaluation of the EF and JR, the countermovement jump was used. In addition to its practicality and specificity with volleyball, the countermovement jump is a widely used method for evaluating the explosive force of lower limbs [5,11,13]. The individual moves from a standing position, with fixed hands on the hip, feet parallel and distanced as the shoulder width and performs the eccentric phase with approximately $90^{\circ}$ flexion of the hip and knee followed by immediate impulse (concentric phase of the movement) with the knees extended throughout the air phase of the movement. To evaluate the EF, the participants performed 3 vertical jumps separated by 15 seconds, being considered for statistical analysis the value of the highest jump. To evaluate the JR, participants performed 15 vertical jumps separated by 3 seconds. For measurement of the jump height, a CEFISE $₫$ branded contact mat was used, and the values were stored in the Jump System Pro software.

\section{Stretching Protocols}

The PNF stretching method by contract-relax technique [17] was used for the posterior musculature of the lower limbs (hamstring and glutes) in the exercise of unilateral bending of hips with knee in extension. In this method, the agonist musculature was passively stretched to a point of discomfort so then the subject performs a maximum voluntary isometric contraction of 5 seconds. So, the physiotherapist would passively stretch the target musculature for 30 or 60 seconds. Four sets were held, separated by 10 seconds of rest, for each leg in a non-alternating form. In Protocol 1 (P1), a 30-second passive stretching was adopted and in Protocol 2 (P2) a 60 -second passive stretching was adopted, both of which were already used in previous studies $[2,5,11,18,19]$. The total stretching time in Protocols 1 and 2 was 280 and 520 seconds, respectively. To ensure a consistent stretching, the exercises were performed by experienced physiotherapists in the PNF technique. 


\section{Statistical analysis}

Table 1:

\begin{tabular}{|c|c|c|c|c|c|c|}
\hline \multicolumn{7}{|c|}{ EF } \\
\hline & Pre & Post & variation in percentage & standard error & effect size & Magnitude \\
\hline Protocol 30s & $39.2 \pm 3.2$ & $38.4 \pm 3.1$ & -2.4 & 1.1 & 0.47 & Small \\
\hline Protocol 60s & $39.9 \pm 3.5$ & $38.6 \pm 3.6$ & -3.3 & 1.2 & 0.44 & Small \\
\hline \multicolumn{7}{|c|}{ JR } \\
\hline Protocol 30s & $36.2 \pm 2.4$ & $35.6 \pm 2.8$ & -2.3 & 1.0 & 0.57 & Small \\
\hline Protocol 60s & $35.7 \pm 3.5$ & $35.4 \pm 3.2$ & -0.9 & 1.1 & 0.19 & Trivial \\
\hline
\end{tabular}

Data are show on average and standard deviation for all variables (Table 1). The regularity of the data was attested by the Shapiro-Wilk test. The T-test was then used with paired samples to compare the values before and after stretching in the EF and RM variables. The level of significance has been established at $5 \%$. The effect size (d) was calculated according to Rhea [20], considering "trivial < 0.35", "small, 0.35-0.80", "moderate, 0.80-1.5" and "large > 1.5 ". The power test was used to observe the power of the sample in each motor tests.

\section{Results}

No differences were identified between the values of the explosive force before and after stretching in Protocols 1 and $2 \mathrm{t}$ (7) $=1.462, \mathrm{p}=0.261$, and t (7) $=1.402, \mathrm{p}=0.302$, respectively. Similarly, there were also no significant differences for muscular resistance located before and after stretching in Protocols 1 and 2, t (7) = 1.542, p $=0.242$, and $\mathrm{t}(7)=0542, \mathrm{p}=0.712$, respectively. Although there were no statistically significant differences, protocols 1 and 2 caused percentage decreases in the explosive force of $-2.4 \%$ and $-3.3 \%$, respectively. As well as in localized muscular resistance, with deficits of $-2.3 \%$ and $-0.9 \%$ in Protocols 1 and 2 , respectively.

\section{Discussion}

The objective of this study was to analyze the acute effect of two PNF protocols in the explosive force and vertical jump resistance of athletes. A reduction of 2.4 and $3.3 \%$ was found in the explosive force after PNF Protocol 1 and 2, respectively. In JR, it was found a reduction of 2.3 and $0.9 \%$ after PNF protocol 1 and 2, respectively. These differences were not statistically significant, showing that the stretching protocols used in this study were not sufficient to interfere with the tested variables.

Regarding to the explosive force, studies that verified the effect of PNF in the vertical jump performance presented very divergent results. Young and Colleagues [15] did not find statistically significant differences in the performance of vertical jumps after PNF. In this study, experienced men in jumping sports performed stretching of the quadriceps, calf and gluteus by contract-relax technique with three repetitions, five seconds of isometric contraction and 15 seconds of passive stretching. Christensen and Colleagues [16] investigated the effects of different types of warming up, including
PNF, in university athletes of various sports modalities and did not find negative effects on vertical jump performance. The hamstring, quadriceps, adductor and flexors were stretched by the contractrelax technique with only one repetition, two seconds of maximum voluntary isometric contraction and five seconds of passive stretching. These authors conclude that this type of stretching can be carried out prior to activities that use the stretch-shortening cycle.

On the other hand, other authors suggest that PNF protocols should be avoided as an integral part of the warm up conducted prior to explosive force activities $[2,13]$. Church and Colleagues [13] have verified significant differences in the performance of the vertical jump of women athletes from various sports modalities after PNF. In this study the contract-relax agonist-contract (CRAC) technique was used with three repetitions, ten seconds of isometric contraction and ten seconds of passive stretching of the quadriceps and hamstring. Also demonstrating negative effects, Bradley and Colleagues [2] performed the contract-relax technique with four repetitions, five seconds of isometric contraction and 30 seconds of passive stretching of the quadriceps, hamstring and plantar flexors and found reduction of the performance of the vertical jump of university men after PNF. As for the divergences of results found in the studies, it can be pointed out the lack of standardization between the protocols employed in the researches. There are differences between the total time of stretching, between the techniques of PNF, the maximum voluntary contraction times of the elongated musculature, the amount of elongated muscle groupings as well as differences between the populations analyzed. This vast number of protocols that present considerable variations in the stretching variables become biases that can collaborate for the different results found. Taking as an example the time of stretching employed, we see that in this study the stretching was carried out for approximately 4 minutes and 30 seconds during Protocol 1 and 8 minutes and 30 seconds during Protocol 2. Young and Colleagues [15] performed stretching for 6 minutes and Christensen and Colleagues [16] performed stretching for approximately 2 minutes and 50 seconds. Bradley and Colleagues [2], who found significant reduction in performance after PNF, performed longer stretching (10 minutes) compared to the studies mentioned above. 
The differences in the manipulations of the aforementioned variables of stretching may cause differences in the effect of stretching in activities involving the stretch-shortening cycle [21]. Moreover, studies were carried out with subjects of different profiles ranging from experienced jumping activities athletes to non-athlete subjects. This profile difference can present different responses in activities requiring rapid force [22]. It is also assumed that another possible explanation for the differences in performance found after stretching is by the number of stretched muscles. The vertical jump is a complex movement involving the major participation of three muscle groups: hamstring, femoral quadriceps and plantar flexors [2] and between the researches there were differences between the stretched muscles. In the present research only, the hip extenders were stretched. In the other studies, it was used more than one stretching exercise that contemplated two to four muscular groups, between plantar flexors, hamstring, femoral quadriceps, gluteus and adductor of the hip.

Considering that the stretching protocols used in this study did not adversely influence the performance of the vertical jump, it is possible to achieve it prior to the training of EF and JR. Although some studies present restrictions regarding doing stretching exercises before explosive force activities, such as jumps and sprints, it seems that the total volume and the amount of muscles involved are more important to consider, since studies with low volume or few muscles involved did not show deleterious effect on performance.

The new finding of this study is that the PNF protocols used did not reduce the vertical jump resistance. Muscular resistance is an important factor for the performance of many sporting activities [10], but few studies have investigated the influence of PNF in this manifestation of force [9] and, to our knowledge, no other work evaluated the effect of PNF on muscular endurance of athletes by means of vertical jump. Some studies that have verified the effect of PNF on muscle resistance in resisted exercises have found a negative effect of the stretching. Barroso and Colleagues [9] evaluated muscular resistance through the number of repetitions performed in the leg press and verified the reduction of muscular resistance of experienced subjects in strength training that previously performed three series of PNF with the hold-relax technique in the three main muscle groups of the exercise. Franco and Colleagues [10] evaluated muscle resistance through the number of repetitions performed in the bench press and also showed that active subjects in resisted training had reduced muscle resistance after PNF, but this study did not mention important variables of this type of stretching, such as maximum voluntary isometric contraction time and the technique employed.

Differently, this study did not find a reduction in JR after PNF protocols. The divergence between the results can be explained by the influence of methodological differences between studies as the form of evaluation of muscular resistance (resistance exercises and vertical jump), the evaluated body segment (upper and lower segment) as well as by difference between the technique and the volume of the stretching employed. More research needs to be carried out in order to better clarify the effect of PNF on muscular resistance in its various forms of evaluation taking into account the specificity of the sport modality. However, due to the evidences available until this moment, one suggestion for coaches and professional who plan exercises bouts for athletes is use few muscles per session to stretch, trying to decrease the possibility of deleterious effect, whereas has more evidences showing that stretching a lot of muscles by a long period prior to high intensity jump activities in sports could cause decrease of performance [2326].

\section{Conclusion}

The study showed that the PNF protocols used did not generate significant negative effects on jump performance, not interfering neither explosive force nor jump resistance of volleyball athletes. In this way, the stretching protocols used in this study can be included during the warming up of athletes without causing damage to the performance of the vertical jump.

\section{References}

1. Peck E, Chomko G, Gaz DV, Farrell AM (2014) The effects of stretching on performance. Current sports medicine reports 13(3): 179-185.

2. Bradley PS, Olsen PD, Portas MD (2007) The effect of static, ballistic, and proprioceptive neuromuscular facilitation stretching on vertical jump performance. Journal of Strength and Conditioning Research 21(1): 223-226.

3. Safran MR, Seaber AV, Garrett WE (1989) Warm-up and muscular injury prevention an update. Sports Medicine 8(4): 239-249.

4. Smith CA (1994) The warm-up procedure: To stretch or not to stretch: A brief review. Journal of Orthopedic \& Sports Physical Therapy 19(1): 12-17.

5. Lam Quang Thanh, Nguyen Thanh Lam (2011) Measurement in sport. Ho Chi Minh University of Sport-HoChiMinh City, Vietnam

6. Rubini EC, Costa AL, Gomes PS (2007) The effects of stretching on strength performance. Sports medicine 37(3): 213-224.

7. Caplan N, Rogers R, Parr MK, Hayes PR (2009) The effect of proprioceptive neuromuscular facilitation and static stretch training on running mechanics. The Journal of Strength \& Conditioning Research 23(4): 1175-1180.

8. Bui Trong Toai (1996) with the topic Research to determine the test system of physical fitness testers young women volleyball. Master thesis, Ho Chi Minh City, VietNam.

9. Barroso R, Tricoli V, dos Santos Gil S, Ugrinowitsch C, Roschel H (2012) Maximal strength, number of repetitions, and total volume are differently affected by static-, ballistic- and proprioceptive neuromuscular facilitation stretching. The Journal of Strength \& Conditioning Research 26(9): 2432- 2437.

10. Franco BL, Signorelli GR, Trajano GS, de Oliveira CG (2008) Acute effects of different stretching exercises on muscular endurance. The Journal of Strength \& Conditioning Research 22(6): 1832-1837.

11. Dao Chanh Thuc (2017) Research and building system developmental strength exercises system by plyometric method for male students' 
major in volleyball of physical education department at An Giang University. International Journal of Physical Education, Sports and Health 4(6): 156-158.

12. Dao Chanh Thuc (2017) Studying and building volleyball curriculum in optional hour at An Giang University: An Giang province. International Journal of Physical Education, Sports and Health 4(6): 137-142.

13. Church JB, Wiggins MS, Moode FM, Crist R (2001) Effect of warm-up and flexibility treatments on vertical jump performance. Journal of strength and conditioning research 15(3): 332-336.

14. Sharman MJ, Cresswell AG, Riek S (2006) Proprioceptive neuromuscular facilitation stretching. Sports Medicine 36(11): 929-939.

15. Young W, Elliott $S$ (2001) Acute effects of static stretching, proprioceptive neuromuscular facilitation stretching, and maximum voluntary contractions on explosive force production and jumping performance. Research quarterly for exercise and sport 72(3): 273-279.

16. Christensen BK, Nordstrom BJ (2008) The effects of proprioceptive neuromuscular facilitation and dynamic stretching techniques on vertical jump performance. The Journal of Strength \& Conditioning Research 22(6): 1826-1831.

17. Alter MJ (2004) Science of flexibility. Human Kinetics.

18. Behm DG, Blazevich AJ, Kay AD, McHugh M (2015) Acute effects of muscle stretching on physical performance, range of motion, and injury incidence in healthy active individuals: a systematic review. Applied physiology, nutrition, and metabolism 41(1): 1-11.
19. Chanh Thuc Dao (2018) Measurement in sports. LAP LAMBERT Academic Publishing.

20. Rhea MR (2004) Determining the magnitude of treatment effects in strength training research through the use of the effect size. Journal of strength and conditioning research 18(4): 918-920.

21. Kallerud H, Gleeson N (2013) Effects of stretching on performances involving stretch-shortening cycles. Sports medicine 43(8): 733-750.

22. Avloniti A, Chatzinikolaou A, Fatouros IG, Avloniti C, Protopapa M, et al. (2016) The acute effects of static stretching on speed and agility performance depend on stretch duration and conditioning level. The Journal of Strength \& Conditioning Research 30(10): 2767-2773.

23. Dao Chanh Thuc, Nguyen Van Tang, Le Thi Minh Dao (2018) Studying and building the developmental strength exercises system by plyometric method for professional female volleyball players. Journal of Physical Education Research 5(1): 01-06

24. Shellock FG, Prentice WE (1985) Warming-up and stretching for improved physical performance and prevention of sports-related injuries. Sports Medicine 2(4): 267-278.

25. Le Quy Phuong (2008) Warm up I. Sports medicine 33(6): 139-154.

26. Nguyen Thanh Lam (1998) Research on development of physical strengths of 15-18-year-old female volleyball players, $\mathrm{PhD}$ thesis on education, Vietnam.

\section{CC) (P) This work is licensed under Creative}

To Submit Your Article Click Here:

Submit Article

DOI: $10.32474 /$ OSMOAJ.2018.02.000132

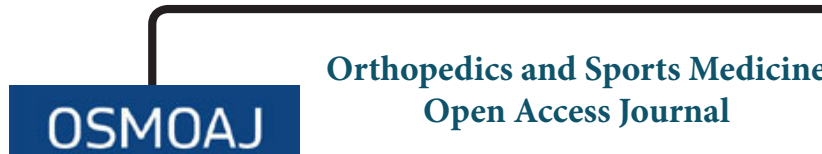

Assets of Publishing with us

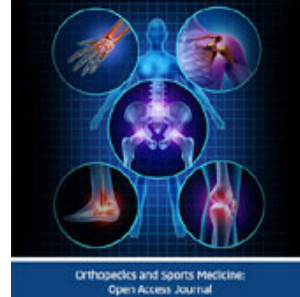

- Global archiving of articles

- Immediate, unrestricted online access

- Rigorous Peer Review Process

- Authors Retain Copyrights

- Unique DOI for all articles 